\title{
Image compression using singular value decomposition by extracting red, green, and blue channel colors
}

\author{
Shamsul Fakhar Abd Gani ${ }^{1}$, Rostam Affendi Hamzah ${ }^{1}$, Ramlan Latip ${ }^{1}$, Saifullah Salam ${ }^{1}$, Fatin \\ Noraqillah $^{1}$, Adi Irwan Herman ${ }^{2}$ \\ ${ }^{1}$ Fakulti Teknologi Kejuruteraan Elektrik and Elektronik, Universiti Teknikal Malaysia Melaka, Melaka, Malaysia \\ ${ }^{2}$ Product and Test Engineering, Texas Instrument (Malaysia), Melaka, Malaysia
}

\begin{tabular}{l} 
Article Info \\
\hline Article history: \\
Received May 5, 2020 \\
Revised May 20, 2021 \\
Accepted Dec 23, 2021
\end{tabular}

Keywords:

Image compression

Image extraction

Image processing

Image reconstruction

Singular value decomposition

\begin{abstract}
This paper presents an image compression using singular value decomposition (SVD) by extracting the red, green, and blue (RGB) channel colors. Image compression is needed in the development of various multimedia computer services and applications for example in the telecommunications and storage technologies. Now a days, video technology, digital broadcast codec and teleconferencing become popular and always requires high image compression process for display. Hence, efficient image compression is compulsory to reduce the number of storage sizes and maintain the image quality. Therefore, this article proposes image compression using SVD, which this method is efficiently reducing the image storage size and at the same time maintaining the image quality. The SVD removes redundant pixel values based on RGB colors to make the storage image size decreased. Based on the experimental analysis on two different type of image extensions (i.e., jpg and png), the SVD is capable to reduce the image size and at the same time preserving the image quality.
\end{abstract}

This is an open access article under the CC BY-SA license.

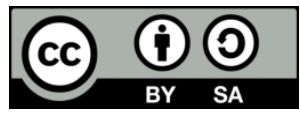

\section{Corresponding Author:}

Rostam Affendi Hamzah

Fakulti Teknologi Kejuruteraan Elektrik and Elektronik, Universiti Teknikal Malaysia Melaka

Hang Tuah Jaya, 76100 Durian Tunggal, Malaysia

Email: rostamaffendi@utem.edu.my

\section{INTRODUCTION}

This article proposes an image compression method to reduce the storage size and maintain the image quality. Currently, images require high data storage for example on high definition display [1], [2]. The important data need an effective method to be stored due to high pixels intensities on images which need a large size of storage and bandwidth of transmission [2]-[4]. Hence, this project is using compression method with singular value decomposition (SVD) technique by using MATLAB software to solve this matter. The SVD is also fast to encode the data (i.e., image in pixel intensity values) and the sequence of transmission is processed effectively [5]. Image compression is only possible technique to remove the redundancy of unwanted pixel or data when the information usually showed in the format which longer than needed [6]. As example, the input data is associated with a certain amount of redundancy. New image compression technologies are being sought everywhere that yield low bit rate while maintaining the fidelity of raw images. Therefore, the transmission of compressed images saves time, cost, and space in long-distance communication [7]-[9].

In this globalization, digital photographs are one of the crucial elements that is important in our life. As we know, the size of an image is quite large because the image size is depending on the number of pixels. Issues such as storage space and the need for rapid transmission data image through the internet and networks 
have resulted in the development of a wide range of image compression techniques to reduce the physical size of files [10]. In such cases, image compression techniques are introduced to decrease quantity data of image to signify exactly or approximately the similar information. The resolution must be fixed as original image while the storage could be reduced at certain ratio [11]. There are few common image formats like bitmap (BMP), joint photographic experts group (JPEG), graphic interchange format (GIF), scalable vector graphic (SVG), portable network graphic (PNG), animated GIF (GIF89a), and tag image file format (TIFF). All these formats are capable to be compressed to make the data size smaller than the original images [12]-[14].

Fundamentally, image compression is a process that uses the minimum bit number to represent the image information with little distortions while ensuring the image quality [15]. The process of image compression is to look for an appropriate encoding or transform method to reduce the data volume which can represent this image. By reducing the redundant data of image, the first step is to compress the data volume of image [16]. The image is stored in a machine system standard form of data matrix; therefore, a series of transform is conducted on that data matrix to reduce the redundant part. The compression method used is rely on the ideal output value [17]. If the application of image compression is predictable to generate an excessive output quality without any losses of fidelity, the method of lossless compression is used while for lossy compression method, it will be used in applications when the quality of the image may be comprised. In this method would have a small loss of quality, but it is not be visible and it also acceptable to make a small compromise on image quality [18].

Nowadays, even though the multimedia such as an image is important in our daily life it also could been a problem to the people. Remote computing has transmitted large-scale information and there is tremendous growth in the progress of massive storage and retrieval systems [19]. To allow a large amounts of data transfer between computers and remote terminals, an additional storage must be installed to cope with the growth in database size. This leads to both cost and equipment being increased. There is one method to solve this matter, which is by using compression process, where it is possible to encode the database and the sequence of transmission effectively [20]. Compression is only possible when the information usually showed in the format which longer than needed. As example, the input data is associated with a certain amount of redundancy. New image compression technologies are being sought everywhere that yield low bit rate while maintaining the fidelity of raw images. Therefore, the transmission of compressed images saves time, cost and space in long-distance communication [21].

This technique of image compression focuses on a large community of people based on the condition required. Firstly, it focuses on the community of people who want to store the image in some quantities of memory card, universal serial bus (USB) flash drive, hard disk, or other storage devices required. Due to this matter, smaller image size, the more image that can be stored in the required storage. Next, this technique also focuses on users who intend to send image data over the internet and download image. "Whatsapp" and "Telegram" is currently the main methods of data transfer. The time to transfer the image will be reduced with smaller size and the uses of the data plan will be minimum. The objective of the article is to compress the redundancy between the pixels by using SVD, to reconstruct the compressed image with smaller storage size and to maintain the image quality before and after the process of compression.

\section{RESEARCH METHOD}

Basically, image compression contains several consecutive processes. The input images could be different type of extensions. In this article, two type of extension have been selected which are the jpg and png. Based on the Figure 1 is being as, it shows the progression of the proposed work from the first to the last. The first step is by selecting and uploading the original input image that need to be compressed. Whether the image types uploaded are correct or not, the system will read the input image. In this article, the types of image that has been selected to be compressed are jpg and png. If other image type has been selected, the user has to re-upload the right original image to avoid error in the system. If the image type is correct, the image will be shown with the original storage size of image with its resolution. Then, the preprocessing stage will extract the red, green, and blue (RGB) components of the image.

The extraction process requires some iterations and pixel modifications on each color channel of the pixel intensities to make the compressed image maintain the original quality. After that, the process of SVD takes place and through the process of computing the SVD for each component by matrices. Each component will be computed through the SVD algorithm which starts with forward transform, quantizer, and encoder. This process eliminates the unnecessary redundancy pixels and relocate pixels of interest each time the SVD computation occurred. Then, a new compressed image will be reconstructed with difference data size and maintain the resolution. If the image cannot be compressed, the process will turn back to the first step for troubleshooting and the user must upload the original image again. 


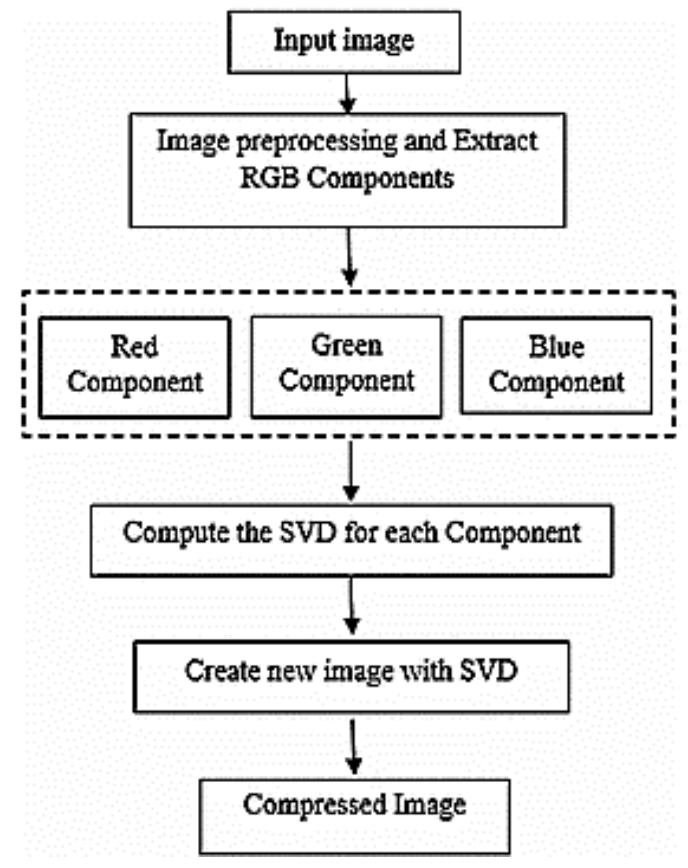

Figure 1. Block diagram and structure of the complete proposed work

Figure 2 shows the development of the image compression technique on the SVD basic block diagram. Original image must be selected as the input image after the program has been executed. Then, RGB channels will be extracted and computed in the forward transform. The quantization step will normalize the resulting matrix, taking the psychovisual properties into account [22], [23]. Next, proceed to the encoder process by simply converting the data from one format to another format or pixel-based format to get better image resolution [24], [25]. Lastly, the compressed image is reconstructed with the same resolution but difference data size. The SVD is developed based on the matrices in image processing. Therefore, (1) of the SVD is being as;

$$
A=U S V^{T}=\sum_{r=1}^{n}\left(\sigma_{r} \times U_{r} \times V_{r}^{T}\right)
$$

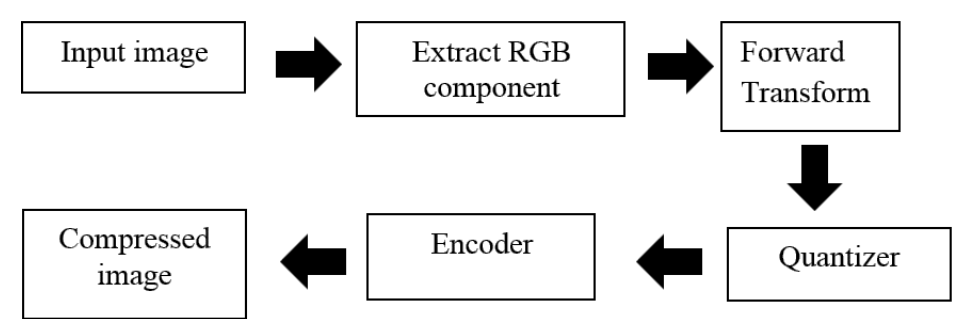

Figure 2. The SVD block diagram implemented in this work

where $A$ is an image matrix of $(m \times n)$ size, $U(m \times m)$ and $V(n \times n)$ denote the unitary matrices, $S=\operatorname{diag}\left(\sigma_{1}, \sigma_{2}, \sigma_{3} \ldots \sigma_{r}\right)$ with the conditions $\sigma_{1}>\sigma_{2}>\sigma_{3}>0$ and $r$ is the rank of matrix $A$. The $\mathrm{S}$ is the singular value the first diagonal terms are positive, all the others were zero, then the $S$ is represented by (2):

$$
S=\left(\begin{array}{ccc}
\sigma_{i} & \cdots & 0 \\
\vdots & \ddots & \vdots \\
0 & \cdots & \sigma_{n}
\end{array}\right)
$$

Then, the image of $I$ in SVD composition is represented by (3):

$$
\|I\|=\operatorname{trace}\left[I^{T} \times I\right]
$$


$=\sum_{i=1}^{m} \sum_{j=1}^{n} I^{2}(i, j)$

$=\sum_{i=1}^{n} \sigma_{i}^{2} \sigma$

In MATLAB running procedures of this project, the flow is given in Table 1 is being as:

Table 1. The procedures applied in the MATLAB

\begin{tabular}{|c|c|}
\hline Steps & Running procedures \\
\hline 1 & Given an input image matrix I \\
\hline 3 & Use $\mathrm{II}^{\mathrm{T}}$ to find the eigenvalues and eigenvectors to form the columns of $\mathrm{U}$ : (II $\left.{ }^{\mathrm{T}}-\kappa \mathrm{I}\right) \ddot{\mathrm{x}}=0$ \\
\hline 5 & Divide each eigenvector by its magnitude to form the columns of $\mathrm{U}$ and $\mathrm{V}$. \\
\hline 6 & $\begin{array}{l}\text { Take the square root of the eigenvalues to find the singular values and arrange them in the diagonal matrix Sin descending } \\
\text { order: } \sigma_{1}>\sigma_{2}>\sigma_{3}>0\end{array}$ \\
\hline
\end{tabular}

\section{RESULTS AND DISCUSSION}

The experimental analysis was conducted using a personal computer with 8G RAM and i5 $3.4 \mathrm{GHz}$ processor. There were two types of image format used in the experimental analyses in this article which are joint photographic group (JPG) and portable network graphic (PNG). Two standard images were used named "woman" and "elephant" with JPG and PNG extensions. Therefore, two different analyses were conducted based on these image extensions. Basically, two difference extension with similar images were used to measure the constancy and strength of the proposed method. The flexibility and complex content structures of images can be revealed the suitability and ability of the proposed work in this article. The first part, JPG images were used with the names women.jpg and elephant.jpg. The experimental analyses and the results are displayed in Figure 3. Based on this figure, the SVD method is capable to reduce the storage of image size from $40.9 \mathrm{~KB}$ to $28 \mathrm{~KB}$ while maintaining the image quality. It takes about $31.5 \%$ of compression ratio for woman.jpg. Additionally, the storage size of elephant.jpg is also decreased about $30 \%$ of ratio as tabulated in Table 2. Figure 3(a) reveals the RGB channels extractions where the quality of the images is improved consequently top to down images due to the increment of intensity values at $(1,10,50$, and 100) respectively. Figure 3(b) displays the original image size at $40.9 \mathrm{~KB}$ while Figure 3(c) is the result after the compression process with the storage size reduction until $28 \mathrm{~KB}$.

Second part, the same images were used but with PNG extension. Figure 4 shows an Elephant.png image. The RGB channels are extracted as shown by Figure 4(a) which the intensity is increased from $(1,10$, 50, and 100) separately. The original image in Figure 4(b) contains $660 \times 371$ resolution and $557 \mathrm{~KB}$ of storage size. The result is displayed in Figure 4(c) where the storage size is decreased from $557 \mathrm{~KB}$ to $401 \mathrm{~KB}$ with $28 \%$ of compression ratio. For the woman.png image, it takes about $23.5 \%$ of compression ratio from $302 \mathrm{~KB}$ to $231 \mathrm{~KB}$. From visualization practice in each figure, the quality of the results is good, and the textures of the images still can be seeing and spotting especially on the edges. Based on the experimental analysis conducted, it validates that the SVD with RGB extraction is capable to reduce the storage size as tabulated in Table 2 at the same time maintaining the image quality. Figure 4(c), the elephant.png structures are clearly displayed with the sky, plants, and the elephant shapes itself. The image color almost similar with the original image before the compression process takes place. It proves that the SVD is capable to preserve the image quality.

The comparison with other methods is also presented as tabulated in Table 2. This quantitative measurement relates to the best achievement for two different types of extension and the ratio before and after the compression process. The comparison is performed based on the lowest storage size and compression ratio for the proposed method with three other published methods in [7], [12], [20]. The lowest storage size for four types of images is the proposed method in this article which uses SVD technique with RGB color extraction. The compression ratios for these images are also high that indicate the proposed work is accurate for all images. The highest compression ratio occurred for the women.jpg image, then followed by elephant.jpg, elephant.png, and woman.png. Second best method is developed in [12] then followed by [7], [20]. The compression ratio for the png images is lower than the jpg images. It occurs for both of the woman.png and elephant.png images respectively.

Figure 5 shows the analysis of root mean square (RMS error) vs Approximation rank $r$ which the rank is explained in (1). This analysis is important to determine the image quality before and after the 
compression process. The woman.jpg and elephant.jpg image were used for this study. Figure 5(a) displays the elephant.jpg result where the RGB color channels indicate more than 35 of RMS error with the rank at 1 intensity value. The RMS error value is decreasing means that the SVD algorithm is capable to maintain the quality at the lowest RMS error for each color channel. Figure 5(b) also shows similar effect for the RMS error which it is more than 35 of RMS error with the rank at 1 intensity value of woman.jpg image. Therefore, based on these two graphs, the quality after the compression process goes to woman image which the image quality is better than elephant image. Additionally, the compression ratio for this image is the highest in this experimental analysis. This indicates that the SVD method by extracting the RGB color channels is able to reduce the storage size and maintain the image quality.

Table 2. Compressed size and ratio of the proposed method and some other methods

\begin{tabular}{cccccc}
\hline Image & Method & Original size $(\mathrm{KB})$ & Resolution & Compressed size (KB) & Compression ratio (\%) \\
\hline women.jpg & Proposed method & 40.9 & $352 \times 440$ & 28 & 31.5 \\
elephant.jpg & Proposed method & 48.1 & $660 \times 371$ & 33.5 & 30 \\
women.png & Proposed method & 302 & $352 \times 440$ & 231 & 23.5 \\
elephant.png & Proposed method & 557 & $660 \times 371$ & 401 & 28 \\
women.jpg & {$[12]$} & 40.9 & $352 \times 440$ & 30 & 25 \\
elephant.jpg & {$[12]$} & 48.1 & $660 \times 371$ & 38 & 21 \\
women.png & {$[12]$} & 302 & $352 \times 440$ & 250 & 21.2 \\
elephant.png & {$[12]$} & 557 & $660 \times 371$ & 470 & 15.6 \\
women.jpg & {$[7]$} & 40.9 & $352 \times 440$ & 35 & 14.4 \\
elephant.jpg & {$[7]$} & 48.1 & $660 \times 371$ & 42 & 13 \\
women.png & {$[7]$} & 302 & $352 \times 440$ & 280 & 7 \\
elephant.png & {$[7]$} & 557 & $660 \times 371$ & 490 & 12 \\
women.jpg & {$[20]$} & 40.9 & $352 \times 440$ & 39 & 2 \\
elephant.jpg & {$[20]$} & 48.1 & $660 \times 371$ & 45 & 6 \\
women.png & {$[20]$} & 302 & $352 \times 440$ & 290 & 8 \\
elephant.png & {$[20]$} & 557 & $660 \times 371$ & 510 & \\
\hline
\end{tabular}
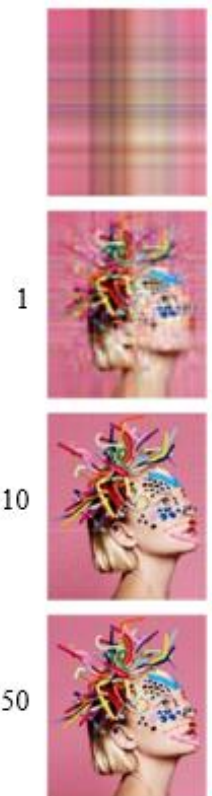

100

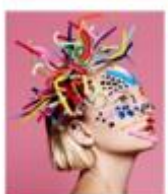

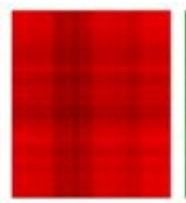
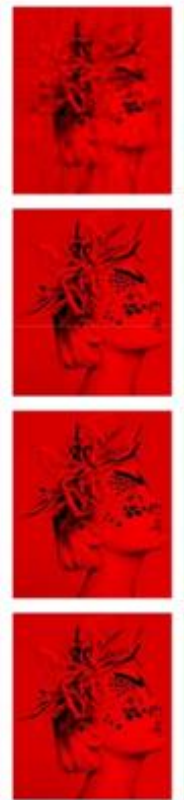

(a)
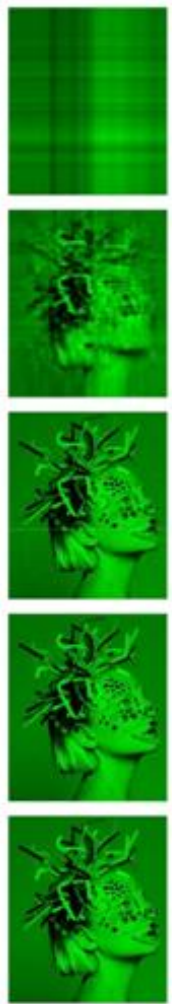
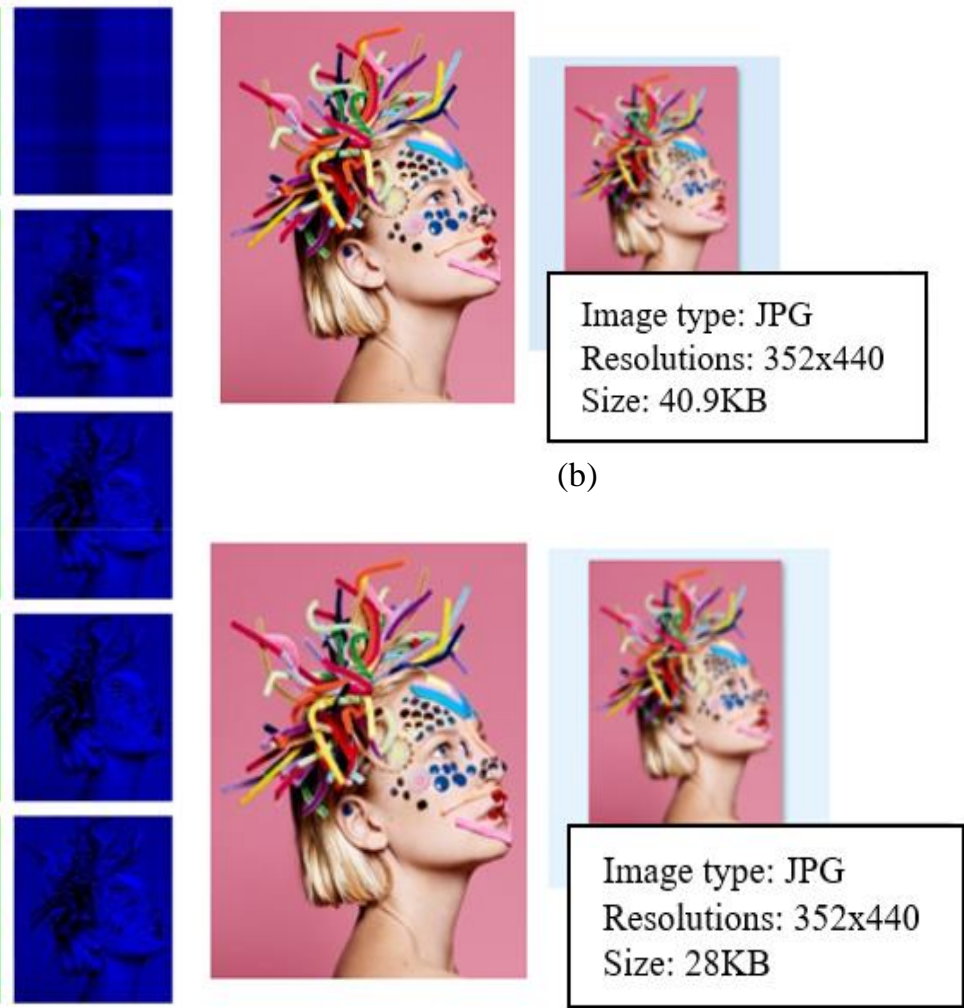

(c)

Figure 3. Women images using JPG extension; (a) set of RGB component values start with (1, 10, 50, and 100), (b) original image with resolution at 352 x 440 and storage size $40.9 \mathrm{~KB}$, and (c) output image with the same resolution and decreased storage size at $28 \mathrm{~KB}$. The image quality is also excellent 

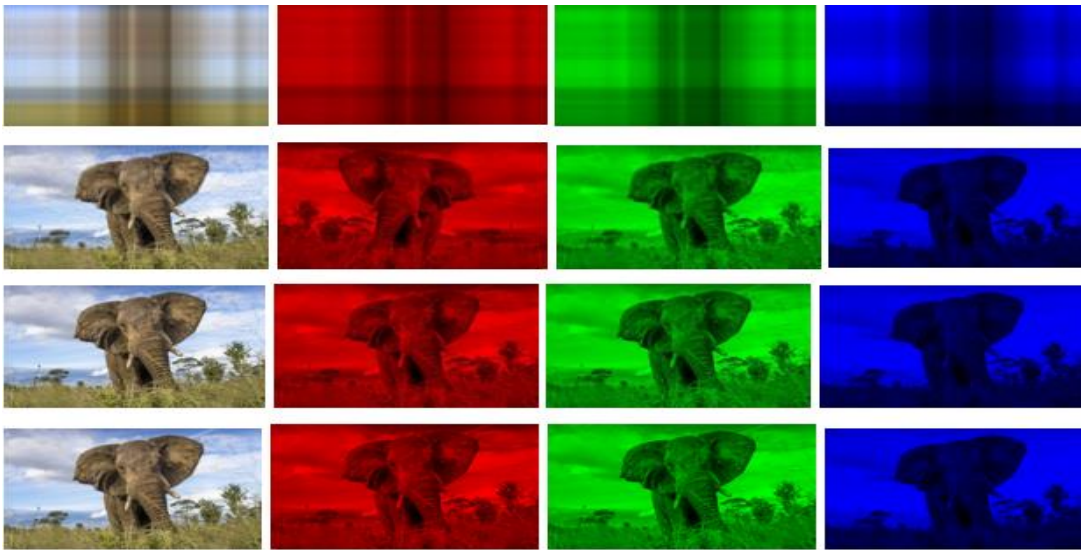

(a)
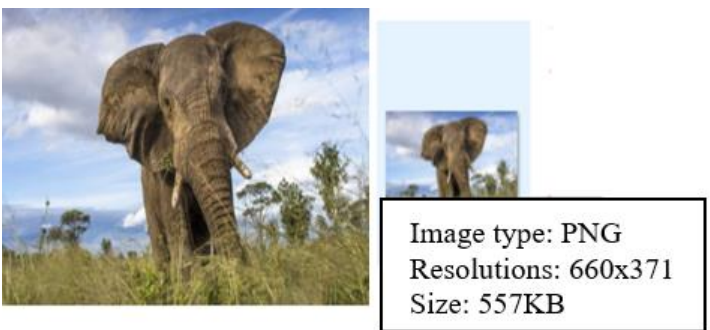

(b)
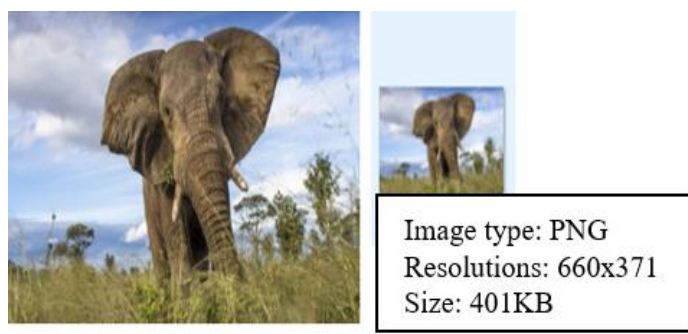

(c)

Figure 4. Elephant images using PNG extension; (a) set of RGB component values start with (1, 10, 50, and $100)$, (b) original image with resolution at $660 \times 371$ and storage size $557 \mathrm{~KB}$ and, (c) output image with the same resolution and decreased storage size at $401 \mathrm{~KB}$

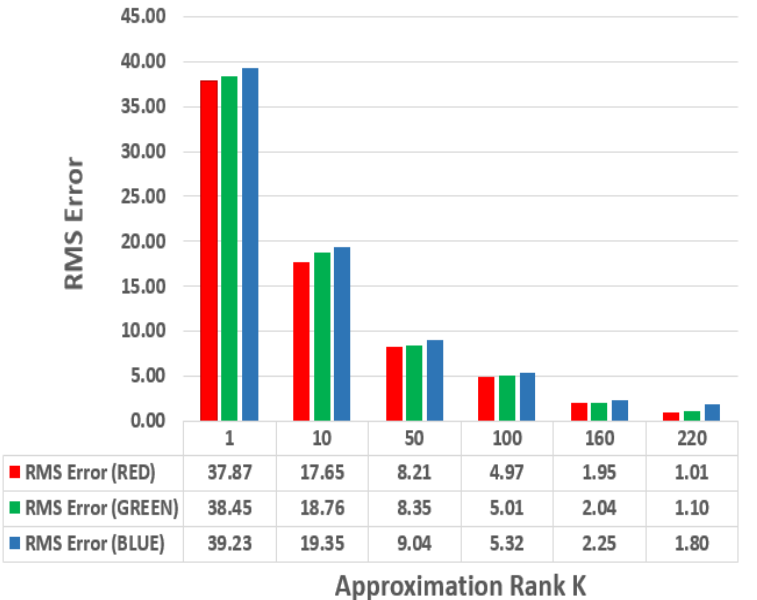

(a)

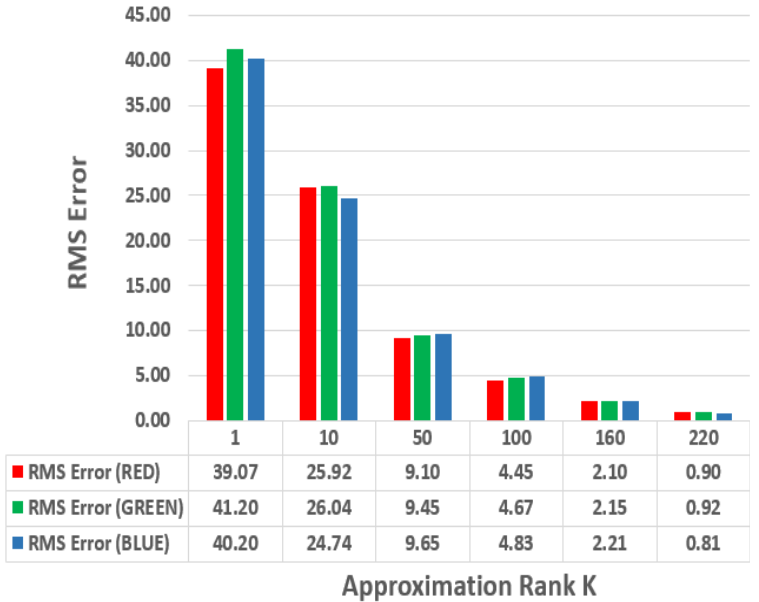

(b)

Figure 5. RMS error vs approximation rank (a) result of elephant.jpg and (b) result of woman.jpg

\section{CONCLUSION}

Image compression is crucial in current storage and display evolution technologies. These technologies have attributes to be treated which require smaller size of image storage and at the same time maintaining the best of image quality. Hence, this article proposed an image compression technique to entertain the respected attributes. Based on the experimental results, the SVD with RGB color extraction is capable to reduce the image size and preserved the image quality. Table 2 tabulates the quantitative results of the proposed method in this article. Additionally, the proposed work is also competitive with some currently published image compression methods. Two different image sizes and extensions have been used in this article and the results shown inspiring ratio and good quality. Without image compression, it would be excessive to have the time and bandwidth to get the information. 


\section{ACKNOWLEDGEMENTS}

This project was supported by the Universiti Teknikal Malaysia Melaka.

\section{REFERENCES}

[1] G. Toderici et al., "Full resolution image compression with recurrent neural networks," in Proceedings of the IEEE Conference on Computer Vision and Pattern Recognition, 2017, pp. 5306-5314, doi:10.1109/CVPR.2017.577.

[2] O. Rippel and L. Bourdev, "Real-time adaptive image compression," in Proceedings of the 34th International Conference on Machine Learning, 2017, pp. 2922-2930, doi: 10.5555/3305890.3305983.

[3] N. Zhou, S. Pan, S. Cheng, and Z. Zhou, "Image Compression-encryption Scheme Based on Hyper-chaotic System and 2D Compressive Sensing," Optics \& Laser Technology, vol. 82, pp. 121-133, Aug. 2016, doi: 10.1016/j.optlastec.2016.02.018.

[4] R. A. Hamzah, M. G. Y. Wei, and N. S. N. Anwar, "Development of Stereo Matching Algorithm based on Sum of Absolute RGB Color Differences and Gradient Matching," International Journal of Electrical and Computer Engineering (IJECE), vol. 10, no. 3, pp. 2375-2381, June 2020, doi: 10.11591/ijece.v10i3.pp2375-2382.

[5] S. Fakhar A. G., M. Saad H., A. Fauzan K., R. Affendi H., and M. Aidil A., "Development of Portable Automatic Number Plate Recognition (ANPR) System on Raspberry Pi," International Journal of Electrical and Computer Engineering (IJECE), vol. 9, no. 3, pp. 1805-1810, June 2019, doi: 10.11591/ijece.v9i3.pp1805-1813.

[6] J. Na'am, J. Harlan, S. Madenda, J. Santony, and C. Suharinto, "Detection of Proximal Caries at the Molar Teeth using Edge Enhancement Algorithm," International Journal of Electrical and Computer Engineering (IJECE), vol. 8, no.5, pp. 3259-3265, October 2018, doi: 10.11591/ijece.v8i5.pp3259-3266.

[7] R. A. Hamzah, M. G. Y. Wei, and N. S. N. Anwar, "Stereo Matching based on Absolute Differences for Multiple Objects Detection," TELKOMNIKA Telecommunication, Computing, Electronics and Control, vol. 17, no. 1, pp. 261-267, February 2019, doi: 10.12928/TELKOMNIKA.v17i1.9185.

[8] Liliana, J. H. Chae, J. J. Lee, and B. G. Lee, “A Robust Method for VR-based Hand Gesture Recognition Using Density-based CNN," TELKOMNIKA Telecommunication, Computing, Electronics and Control, vol. 18, no. 2, pp. 761-769, April 2020, doi: 10.12928/TELKOMNIKA.v18i2.14747.

[9] A. J. Hussain, A. Al-Fayadh, and N. Radi, "Image Compression Techniques: A survey in Lossless and Lossy Algorithms," Neurocomputing, vol. 300, pp. 44-69, July 2018, doi: 10.1016/j.neucom.2018.02.094.

[10] J. Zhao, P. An, X. Huang, C. Yang, and L. Shen, "Light Field Image Compression via CNN-Based EPI Super-Resolution and Decoder-Side Quality Enhancement," in IEEE Access, vol. 7, pp. 135982-135998, 2019, doi: 10.1109/ACCESS.2019.2930644.

[11] L. Gong, K. Qiu, C. Deng, and N. Zhoua, "An Optical Image Compression and Encryption Scheme based on Compressive Sensing and RSA Algorithm," Optics and Lasers in Engineering, vol. 121, pp 169-180, October 2019, doi: 10.1016/j.optlaseng.2019.03.006.

[12] R. A. Hamzah, H. Ibrahim, and A. H. A. Hassan, "Stereo Matching Algorithm based on Illumination Control to Improve the Accuracy,” Image Analysis \& Stereology, vol. 35, no. 1, pp. 39-52, 2016, doi: 10.5566/ias.1369.

[13] N. H. Ja'afar, and A. Ahmad, "Algorithm development and hardware implementation for medical image compression system: review," Indonesian Journal of Electrical Engineering and Computer Science, vol. 18, pp. 1331-1341, 2020, doi: 10.11591/ijeecs.v18.i3.pp1331-1341

[14] S. K. Roy et al., "Fractal Image Compression using Upper Bound on Scaling Parameter, "Chaos, Solitons \& Fractals, vol. 106, pp. 16-22, 2018, doi: 10.1016/j.chaos.2017.11.013.

[15] A. Artusi et al., "Overview and Evaluation of the JPEG XT HDR Image Compression Standard," Journal of Real-Time Image Processing, vol. 16, pp. 413-428, 2019, doi: 10.1007/s11554-015-0547-x.

[16] X. J. Tong et al., "A Joint Color Image Encryption and Compression Scheme based on Hyper-chaotic System, "Nonlinear Dynamics, vol. 84, pp. 2333-2356, 2016, doi: 10.1007/s11071-016-2648-X.

[17] S. Liu et al., "A Fractal Image Encoding Method based on Statistical Loss used in Agricultural Image Compression," Multimedia Tools and Applications, vol. 75, pp. 15525-15536, 2016, doi: 10.1007/s11042-014-2446-8.

[18] K. Kurihara et al., "An Encryption-then-compression System for Lossless Image Compression Standards, "IEICE Transactions on Information and Systems, vol. 100, pp. 52-56, 2017, doi: 10.1587/transinf.2016MUL0002.

[19] Y. Zhang et al., "A Novel Image Compression-encryption Hybrid Algorithm based on the Analysis Sparse Representation," Optics Communications, vol. 392, pp. 223-233, 2017, doi: 10.1016/j.optcom.2017.01.061.

[20] R. A. Hamzah et al., "Improvement of Stereo Matching Algorithm based on Sum of Gradient Magnitude Differences and Semiglobal Method with Refinement Step, “Electronics Letters, vol. 54, pp. 876-878, 2018, doi: 10.1049/el.2017.3956.

[21] M. T. Bennani et al., "A New Leach Protocol based on ICH-Leach for Adaptive Image Transferring using DWT," TELKOMNIKA Telecommunication, Computing, Electronics and Control, vol. 17, no. 5, pp. 2418-2426, October 2019, doi: 10.12928/TELKOMNIKA.v17i5.12446.

[22] C. Raghavendra, S. Sivasubramanian, and A. Kumaravel, "Improved Image Compression using Effective Lossless Compression Technique,” Cluster Computing, vol. 22, no. 2, pp. 3911-3916, 2019, doi: 10.1007/s10586-018-2508-1.

[23] L. Gong, K. Qiu, C. Deng, and N. Zhoua, "An Image Compression and Encryption Algorithm based on Chaotic System and Compressive Sensing," Optics \& Laser Technology, vol. 115, pp. 257-267, July 2019, doi: 10.1016/j.optlastec.2019.01.039.

[24] N. Zhou, H. Jiang, L. Gong, and X. Xie, "Double-image Compression and Encryption Algorithm based on Co-sparse Representation and Random Pixel Exchanging," Optics and Lasers in Engineering, vol. 110, pp. 72-79, Nov. 2018, doi: 10.1016/j.optlaseng.2018.05.014.

[25] M. Li, K. Ma, J. You, D. Zhang, and W. Zuo, "Efficient and Effective Context-Based Convolutional Entropy Modeling for Image Compression," in IEEE Transactions on Image Processing, vol. 29, pp. 5900-5911, 2020, doi: 10.1109/TIP.2020.2985225. 


\section{BIOGRAPHIES OF AUTHORS}
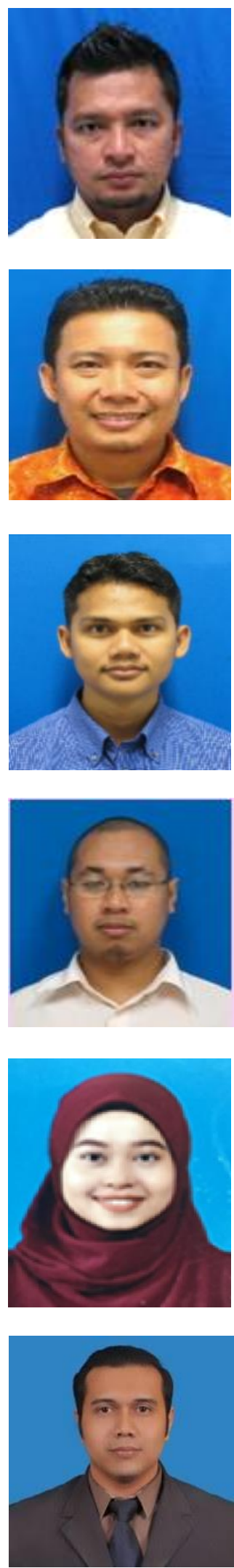

Shamsul Fakhar Abd Gani (D) 8d SC P graduated from Universiti Malaysia Perlis (UniMAP) in Bachelor of Engineering (Computer Engineering) with honours in 2006 and later received his Master's degree in Internet \& Web Computing in 2015 from Royal Melbourne Institute of Technology (RMIT) Australia. He started his career as an R\&D electronic engineer specializing in software design for meter cluster development in Continental Automotive Malaysia. He is currently a lecturer in electronic and computer engineering technology department in Universiti Teknikal Malaysia Melaka (UTeM). He can be contacted at email: shamsulfakhar@utem.edu.my.

Rostam Affendi Hamzah Hamzah (D) 81 sC P graduated from Universiti Teknologi Malaysia where he received his B.Eng majoring in Electronic Engineering. Then he received his M. Sc. majoring in Electronic System Design Engineering and $\mathrm{PhD}$ majoring in Electronic Imaging from the Universiti Sains Malaysia. Currently he is a lecturer in the Universiti Teknikal Malaysia Melaka teaching digital electronics, digital image processing and embedded system. He can be contacted at email: rostamaffendi@utem.edu.my.

Ramlan bin Latip (D) 81 SC P graduated from Universiti Teknologi Malaysia, with B. Eng. (Electrical Engineering) in 2006. He received his MSc. in Electrical Engineering from University of Nottingham, United Kingdom in 2016. From year 2007 until 2011 he involved in electronic industry and oil \& gas industry as an electrical engineer. He is currently work as Senior Teaching Engineer and Lab Manager at Faculty of Electrical and Electronic Engineering Technology, Universiti Teknikal Malaysia Melaka. His research interest includes process control system, sensors and transducers and energy efficiency. He can be contacted at email: ramlan@utem.edu.my.

Saifullah bin Salam (D) IS SC P graduated from Universiti Teknologi Malaysia in Bachelor of Electrical Engineering and finished his Master of Engineering (Industrial Elektronic and Control) from Universiti Malaya, in 2013. He is currently a senior Teaching Engineer attached to Electronic and Computer Engineering Technology Department, Faculty of Electrical and Electronic Engineering Technology, UTeM. He can be contacted at email: saifullah@utem.edu.my.

Fatin Noraqillah (iD SC S Praduated in 2020 with a Bachelor Degree in Electronic Engineering Technology (Telecommunications) from the Universiti Teknikal Malaysia Melaka. Her current research interests are communication, networking, and digital signal processing. She can be contacted at email: b071610360@ student.utem.edu.my.

Adi Irwan Herman (iD 88 SC P graduated in 2015 with a Bachelor Degree in Computer Engineering Technology (Computer System) from the Universiti Teknikal Malaysia Melaka. Currently, he works with Texas Instrument more than 5 years with his current research interests are computer engineering related field of studies. He can be contacted at email: adiirwanherman@gmail.com. 